\title{
Applications of Fourier transform infrared spectroscopy, Fourier transform infrared microscopy and near-infrared spectroscopy to cancer research
}

\author{
Kazuyuki Yano ${ }^{\mathrm{a}, *}$, Yasushi Sakamoto ${ }^{\mathrm{b}}$, Narumi Hirosawa ${ }^{\mathrm{b}}$, Shouko Tonooka ${ }^{\mathrm{b}}$, \\ Hiroo Katayama ${ }^{\mathrm{c}}$, Kuniyoshi Kumaido ${ }^{\mathrm{d}}$ and Akira Satomi ${ }^{\mathrm{e}}$ \\ ${ }^{a}$ Department of Chemistry, Saitama Medical School, 981 Kawakado, Moroyama, Iruma-gun, \\ Saitama 350-0496, Japan \\ ${ }^{\mathrm{b}}$ Department of Biomedical Research Center, Saitama Medical School, 38 Morohongo, Moroyama, \\ Iruma-gun, Saitama 350-0495, Japan \\ ${ }^{\mathrm{c}}$ Saitama Medical College, Saitama Medical School, 38 Morohongo, Moroyama, Iruma-gun, \\ Saitama 350-0495, Japan \\ ${ }^{\mathrm{d}}$ Department of Neurological Surgery, Saitama Medical School, 38 Morohongo, Moroyama, \\ Iruma-gun, Saitama 350-0495, Japan \\ ${ }^{\mathrm{e}}$ Second Department of Surgery, Saitama Medical School, 38 Morohongo, Moroyama, Iruma-gun, \\ Saitama 350-0495, Japan
}

\begin{abstract}
Glycogen levels in human lung and colorectal cancerous tissues were measured by the Fourier transform (FT-IR) spectroscopic method. Reliability of this method was confirmed by chemical analyses of the same tissues used for the FT-IR spectroscopic measurements, suggesting that this spectroscopic method has a high specificity and sensitivity in discriminating human cancerous tissues from noncancerous tissues. The glycogen levels in the tissues were compared with the clinical, histological and histopathological factors of the cancer, demonstrating that glycogen is a critical factor in understanding the biological nature of neoplastic diseases. Furthermore, direct measurement of a very small amount of tissue by a FT-IR microscope suggested that it could be used as a diagnostic instrument for various tissue samples obtained via a fine needle biopsy procedure. The progressive alterations in rat mammary gland tumors were investigated by a near-infrared (NIR) spectrometer with a fiber optic probe. A lipid band due to the first overtone of $n$-alkane was used to quantitatively evaluate malignant changes in the tumors. NIR spectroscopy may offer the potential for non-invasive, in vivo diagnosis of human cancers.
\end{abstract}

\section{Introduction}

Infrared radiation is divided into three regions, 14,000 to $4,000 \mathrm{~cm}^{-1}$ (714 to $2500 \mathrm{~nm}$ ) for nearinfrared, 4000 to $400 \mathrm{~cm}^{-1}$ (2.5 to $25 \mu \mathrm{m}$ ) for mid-infrared, and 400 to $4 \mathrm{~cm}^{-1}$ (25 to $2500 \mu \mathrm{m}$ ) for far-infrared [1]. Most commonly, the mid-infrared radiation is used for infrared spectroscopy because the vast majority of molecules in the universe absorb its light. Fourier transform infrared (FT-IR) spectroscopy, which is a non-invasive physical method, has received attention for investigation of biomolecules and for understanding the biological nature of neoplastic diseases [2-5]. Recently, Fourier

\footnotetext{
*Corresponding author. Tel./Fax: +81 49295 8264; E-mail: kazkyano@ saitama-med.ac.jp.
} 
transform microscopy (FT-IR-MC) has received a great amount of attention from various aspects since it allows highly precious analysis of very small sections of tissue in situ without extraction [6-8]. Diagnostic applications of the mid-infrared spectroscopy, however, are limited because many biological materials absorb the mid-infrared radiation so strongly that the path lengths greater than 10-15 $\mu \mathrm{m}$ are not used. In contrast, near-infrared (NIR) spectroscopy due to scattering phenomena rather than absorption phenomena overcomes this problem and has recently been employed in various fields [9,10]. We would like to review applications of these apparatus to cancer research through our works.

\section{FT-IR spectroscopic study of human lung and colorectal cancers}

Glycogen in malignant cells is generally considered to be a specific differentiation material [11]. We studied glycogen levels in human lung tissues in relation to the histological classification and differentiation of carcinomas by an attenuated total reflectance FT-IR spectroscopic method [4]: A tissue sample $(100 \mathrm{mg})$ was pulverized under liquid nitrogen using a small pre-cooled mortar and pestle. The resulting powder was placed equally on a zinc selenide prism, covered with an aluminum foil, and set on a horizontal attenuated total reflectance accessory. The spectra were obtained with a System 2000FT-IR (Perkin Elmer, Ltd., UK) equipped with a DTGS (deuterated triglycine sulfate) detector.

To quantitatively evaluate glycogen levels in the tissues, we chose the band at $1045 \mathrm{~cm}^{-1}$ that is assigned as the $\mathrm{C}-\mathrm{O}$ stretching coupled with $\mathrm{C}-\mathrm{O}$ bending of the $\mathrm{C}-\mathrm{OH}$ group of glycogen and the band at $1545 \mathrm{~cm}^{-1}$ due to amide II of protein as an internal standard peak, and measured their integrated areas by least-square iterative curve fitting to fit Lorentzian line shapes. From these data, we calculated the ratio of the glycogen band area (A1045) and the protein band area (A1545), and compared these ratios (A1045/A1545) with the clinical and pathological data. The results suggested that the glycogen levels in adenocarcinoma and squamous cell carcinoma were significantly higher than those in the corresponding normal sections and that the relative amount of glycogen in the adenocarcinoma (compared to glycogen level in the corresponding normal section) was significantly higher than that in the squamous cell carcinoma. When the change in the glycogen levels was compared with the different grade of differentiation of squamous cell carcinomas, we found that the glycogen levels in the well-differentiated squamous cell carcinomas were significantly higher than those in poorly differentiated ones.

To find out correlation between the amounts of glycogen measured by our FT-IR spectroscopic method and that by usual chemical methods, we analyzed the glycogen levels in the same tissues as used for FTIR measurements by a chemical method [12] and the protein levels by a dot METRIC (Geno Technology, Inc. St. Louis, USA). The ratio of glycogen levels in the malignant and normal tissues with adenocarcinoma, which were obtained by the FT-IR spectroscopic method, was 2.11 while that by the chemical method was 2.03 . With squamous cell carcinoma, those ratios were 1.63 and 1.51 , respectively. The protein levels were very similar between the malignant and normal tissues in both carcinomas. The results are summarized in Table 1 (unpublished results). From these results, we confirmed that our FT-IR spectroscopic method is very reliable to estimate glycogen levels in human lung tissues.

Next, we investigated the amounts of glycogen in three sections of human colorectal tissues, cancerous sections, adjacent sections and normal sections, by the FT-IR spectroscopic method [5]. Glycogen levels in both the colon and rectum were found to be highest in the cancerous sections, lower in the normal sections and lowest in the adjacent sections. The glycogen level in the rectum, however, seemed to be less than that in the colon. These results were compared with histopathological factors (tumor size and stage of disease) and cell cycle progression. We found that glycogen levels in the cancerous tissues were 
Table 1

Amounts of glycogen and protein in malignant and normal tissues of human lung cancers by chemical methods

\begin{tabular}{lccccccc}
\hline & \multicolumn{3}{c}{ Adenocarcinoma } & & \multicolumn{3}{c}{ Squamous cell carcinoma } \\
\cline { 2 - 4 } \cline { 6 - 8 } & Malignant & Normal & Malignant/Normal & & Malignant & Normal & Malignant/Normal \\
\hline A1045/A1545 $(\%)^{1}$ & 5.58 & 2.65 & 2.11 & & 5.14 & 3.15 & 1.63 \\
Glycogen $(\mu \mathrm{g} / \mathrm{mg} \text { tissue })^{2}$ & 1.06 & 0.52 & 2.03 & & 1.15 & 0.76 & 1.51 \\
Protein $(\mu \mathrm{g} / \mathrm{mg} \text { tissue })^{2}$ & 108 & 106 & 1.02 & & 134 & 129 & 1.04 \\
Glycogen/Protein $(\mu \mathrm{g} / \mathrm{mg})^{2}$ & 9.81 & 4.91 & 2.00 & & 8.58 & 5.89 & 1.46 \\
\hline
\end{tabular}

The values are averages $\left({ }^{1} n=10,{ }^{2} n=5\right)$. Normal lung tissue: A1045/A1545(\%) $=4.25 \pm 0.75(n=5)$.

negatively correlated with the tumor size and the progression of disease stage. In regard to the cell cycle progression, there was a positive correlation between the glycogen level and the proportion of cancer cells in the $G_{1}$ phase while there was a negative correlation between the glycogen level and those in the $\mathrm{S}$ and $\mathrm{G}_{2} \mathrm{M}$ phases. Glycogen levels were highest in the cancers with a high proportion of cells in the $\mathrm{G}_{1}$ phase and decreased with progression to the $\mathrm{S}$ phase. These findings indicate that glycogen may be utilized in synthesis of DNA and that the cancerous tissues are supplied with glycogen from their adjacent tissues. The result is very interesting therapeutically since colorectal cancer growth may be inhibited by artificial control of glycogen level in the $\mathrm{G}_{1}$ phase of cancer cells.

\section{FT-IR microscopic study of human lung cancer}

The FT-IR technique described in the above papers is limited because it requires pretreatment of the samples as well as a quite large amount of the tissue. Furthermore, the spectra themselves do not always provide detailed information on the cancer because of the homogenized samples for FT-IR spectroscopic measurements. Thus, we studied again human lung cancerous and noncancerous tissues by using a FTIR microscope [8]: Tissue samples were embedded in OCT (Optimal Cutting Temperature) and frozen immediately in liquid nitrogen. Each frozen tissue was cut into two successive 10- $\mu$ m-thick sections. One was mounted on a calcium fluoride $\left(\mathrm{CaF}_{2}\right)$ window for FT-IR spectroscopic measurements and the other was fixed on a microscope slide for histopathological examination. Infrared spectra of the dried tissues on the $\mathrm{CaF}_{2}$ window were measured in the frequency region $950-4000 \mathrm{~cm}^{-1}$ at room temperature with a System 2000 FT-IR equipped with an AutoIMAGE Microscope. Each background spectrum was measured at the position of the $\mathrm{CaF}_{2}$ window without a tissue. The spectra were collected with an aperture of $100 \times 100 \mu \mathrm{m}, 50$ scans, and a resolution of $4 \mathrm{~cm}^{-1}$.

Cancerous and noncancerous sections were determined by comparing with the photomicrographs obtained from the hematoxylin-eosin-stained tissues. The spectral patterns in the cancerous sections were quite different from those in the corresponding noncancerous sections. In the frequency region 1430 $1480 \mathrm{~cm}^{-1}$, the band intensities in the cancerous sections were clearly reduced, while in the frequency region $980-1150 \mathrm{~cm}^{-1}$, they were increased when compared with those in the noncancerous sections. Since it has been known that amounts of DNA [6], glycogen and cholesterol [13] in the lung cancerous tissues differ from those in the normal tissues, we chose the bands at $1084 \mathrm{~cm}^{-1}$ (DNA), $1045 \mathrm{~cm}^{-1}$ (glycogen), $1467 \mathrm{~cm}^{-1}$ (cholesterol) and $1545 \mathrm{~cm}^{-1}$ (protein as an internal standard peak), and measured their corresponding corrected peak heights, H1084, H1045, H1467, and H1545, respectively. The amounts of DNA and glycogen in the tissues were expressed as the ratios of H1084/H1545 and H1045/H1545. Furthermore, if decreasing amount of cholesterol has a critical factor for forming the lung cancer, the H1045/H1467 ratio might be more sensitive and specific to the malignant changes than the H1045/H1545 
ratio. These ratios in the cancerous and noncancerous sections indicated that the H1045/H1467 ratio is an exceptionally useful factor for discriminating the cancerous tissues from the noncancerous tissues.

For the microscopic mapping, a square region $(200 \times 200 \mu \mathrm{m})$ of a tissue was selected and mapped with $10-\mu \mathrm{m}$ steps in the $X$ and $Y$ directions (aperture $40 \times 40 \mu \mathrm{m}$ ). The maps were presented by a single wavenumber $\left(1045 \mathrm{~cm}^{-1}\right)$ map using the wavenumber $1467 \mathrm{~cm}^{-1}$ as a reference (Fig. 1). Cancerous sections and noncancerous section were determined by comparing with the photomicrographs obtained from the hematoxylin-eosin-stained tissues. The distribution pattern of the cancerous sections was al-
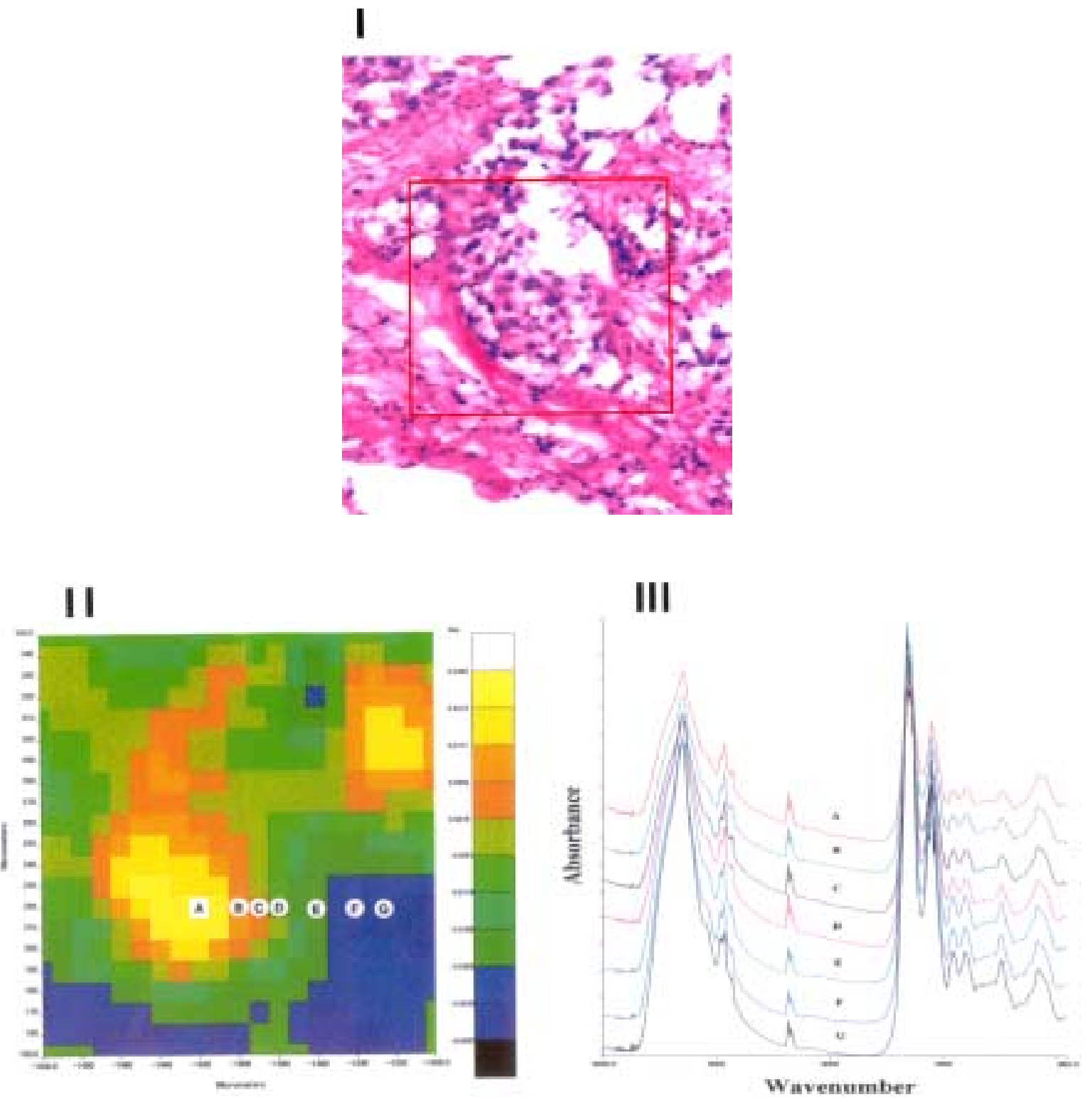

Fig. 1. Photomicrograph $(\times 200$, original magnification $)$ of adenocarcinoma (I), its infrared microscopic map (II), and spectra (III) of the different color clusters on II. 
most exactly the same as that in the corresponding photomicrograph. The spectra of the different color clusters indicated successive changes in the absorptions due to glycogen and cholesterol. These findings demonstrated that the map reflected small changes in the spatial distribution of cancer cells.

To get information on the possibility that FT-IR-MC could be used as a diagnostic instrument, we investigated very small amounts of tissues that might be obtained via a fine needle biopsy procedure using the FT-IR microscope. For the direct measurement, a sample (less than $0.1 \mathrm{mg}$ ) was directly placed on a small $\mathrm{CaF}_{2}$ disk and carefully stretched it with a tweezers and a surgical knife to get the clear or thin section of the tissue. The $\mathrm{H} 1045 / \mathrm{H} 1467$ ratios of the cancerous and noncancerous sections were analyzed as mentioned above. These results were compared with those obtained from the microtomecut slices. The graphic patterns of the direct measurements are very similar to those obtained from the measurements of microtome-cut slices, demonstrating that this simple method will be reliable for the analysis of biopsy samples.

\section{In vivo NIR spectroscopic study of rat mammary gland tumors}

Breast cancer is the most common cancer in women of the western world, and incident rates are constantly increasing even in other countries. In order to get fundamental information on progressive alterations in breast tissues during cancer development, we examined the NIR spectral changes of mammary gland tissues in the carcinogenic processes of rats treated with 7,12-dimethylbenz[a]anthracene (DMBA) [10]. Female Sprague-Dawley rats were treated with $30 \mu \mathrm{l}$ of pentobarbital/kg body wt, and hairs on the measurement sites were removed under anesthesia with a hair clipper. An animal was set on the apparatus, and the fiber optic probe was attached to a clamp to make its tip contact the center of the cancer or the teats of the breast. The contact pressure was adjusted to be as constant as possible by carefully turning the knob of a laboratory jack. Spectral measurements were carried out with a Bruker IFS28/N-spectrometer equipped with a N261 fiber optic probe at $25^{\circ} \mathrm{C}$.

Each spectrum was obtained from 50 interferograms with a resolution of $32 \mathrm{~cm}^{-1}$. The measured reflectance spectra were converted to $\log (1 /$ reflectance $)$ spectra and the spectral wavenumbers $\left(\mathrm{cm}^{-1}\right)$ were converted to wavelengths (micrometers or nanometers). The $\log (1 /$ reflectance) spectra were converted to the second derivative spectra, as the second derivative techniques are usually used in such analyses of NIR spectra to minimize baseline offsets and to resolve overlapping absorption.

Since correlations between NIR spectra and band assignments are not well established in the NIR regions for most compounds, we measured the second derivative spectra of the standard samples (cholesterol, linoleic acid, protein, collagen, elastin and DNA) to obtain information on their characteristic bands in the spectra of mammary gland tissues. The results indicated that the intensities of DNA bands and water bands in the cancerous tissues were relatively increased while those of the lipid bands were reduced in relation to those in the normal site. With protein bands, however, their intensities differed little between both sites. Thus, a lipid band around $1725 \mathrm{~cm}^{-1}$ due to the first overtone of $n$-alkane [3] and a protein band around $2054 \mathrm{~cm}^{-1}$ due to a combination band of amide A and amide II of polypeptides [14] as an internal reference band were chosen for the quantitative evaluation of malignant changes in mammary gland tissues. Using the relative intensities of the second derivative spectra, the lipid/protein ratios were calculated from the spectra of the mammary gland tissues in the control animals and those of noncancerous and cancerous sites in the treated animals. The ratios were significantly different from each other, demonstrating that the lipid concentrations in different mammary gland tissues can be accurately estimated. 
To investigate progressive alterations in the malignant tissues, the second derivative spectral changes were investigated in the 1600 to 1800 -nm region with regard to the elapsed times until 11 weeks after the administration of DMBA. With the cancer growth, the intensity of the lipid band decreased and this band was shifted to higher wavelengths by $7 \mathrm{~nm}$ at the 11 th week compared to the wavelength of the band at the 5th week. The reduced amounts of lipids in the cancerous sites may be caused by a high metabolic demand of lipids in the malignant tumors. Furthermore, the higher wavelength shift may be caused by the formation of elastic fibers in the lipid layer with collagen induced in the cancer tissues.

Histological information on the same tissues as those used for the NIR spectroscopic measurements was necessary to confirm the present spectral data. Thus, we stained the normal and cancerous tissue sections with Masson trichrome dye: The photomicrographs indicate a large difference between the normal and cancerous tissue components: The cancer cells infiltrated into the subcutaneous tissue (mainly lipids) resulting in a marked decrease in tissue thickness around the cancer when compared to normal subcutaneous tissue. In addition, many collagen fibers can be seen in the subcutaneous tissue of the cancer, supporting the NIR spectral findings as mentioned above. These findings are in good agreement with the reduced amounts of lipids and the higher wavelength shifts of the lipid band, which were observed in the NIR spectra in the cancerous tissue. Thus we can get accurate information on changes in whole tissue components by measuring NIR spectra.

\section{Conclusion}

The FT-IR spectroscopic method has a high specificity and sensitivity in discriminating human cancerous tissues from noncancerous tissues. The FT-IR microscope could be used as a diagnostic instrument for various tissue samples obtained via a fine needle biopsy procedure. NIR spectroscopy may offer the potential for non-invasive, in vivo diagnosis of some human cancers. Its application to humans, however, is far more difficult, and much work remains to be done from various aspects.

\section{References}

[1] B. Smith, Infrared Spectral Interpretation. A Systematic Approach. CRC Press, Boca Raton, Florida, 1999, p. 4.

[2] B. Rigas, S. Morgello, I.S. Goldman and R.T.T. Wong, Human colorectal cancers display abnormal Fourier-transform infrared spectra, Proc. Natl. Acad. Sci. USA 87 (1990), 8140-8144.

[3] M. Jackson and H.H. Mantsch, in: Infrared Spectroscopy of Biological Molecules, H.H. Mantsch and D. Chapman, eds, Wiley-Liss, New York, 1996, pp. 311-340

[4] K. Yano, S. Ohoshima, Y. Shimizu, T. Moriguchi and H. Katayama, Evaluation of glycogen level in human lung carcinoma tissues by an infrared spectroscopic method, Cancer Lett. 110 (1996), 29-34.

[5] S. Takahashi, S. Satomi, K. Yano, H. Kawase, T. Tanimizu, Y. Tsuji, S. Murakami and R. Hirayama, Evaluation of glycogen levels in human colorectal cancer tissue: relationship with cell cycle and tumor outgrowth, J. Gastroenterol. 34 (1999), 474-480.

[6] E. Benedetti, L. Teodori, M.L. Trinca, P. Vergamini, F. Salvati, F. Mauro and G. Spremolla, A new approach to the study of human solid tumor cells by means of FT-IR microscopy, Appl. Spectrosc. 8 (1990), 1276-1280.

[7] D.L. Wetzel and S.M. LeVine, Imaging molecular chemistry with infrared microscopy, Science 285 (1999), 1224-1225.

[8] K. Yano, S. Ohoshima, Y. Gotou, K. Kumaido, T. Moriguchi and H. Katayama, Direct measurement of human lung cancerous and noncancerous tissues by Fourier transform infrared microscopy: Can an infrared microscope be used as a clinical tool?, Anal. Biochem. 287 (2000), 218-225.

[9] M.L. McKelvy, T.B. Britt, B.L. Davis, J.K. Gillie, A. Lentz, A. Leugers, R.A. Nyquist and C.L. Putzig, Anal. Chem. 68 (1996), 93R-160R.

[10] N. Hirosawa, Y. Sakamoto, H. Katayama, S. Tonooka and K. Yano, In vivo investigation of progressive alterations in rat mammary gland tumors by near-infrared spectroscopy, Anal. Biochem. 305 (2002), 156-165. 
[11] T. Ito, Y. Noguchi, N. Udaka, H. Kitamura and S. Satoh, Glucose transporter expression in developing fetal lungs and lung neoplasms, Histol. Histopathol. 14 (1999), 895-904.

[12] E. van Handel, Estimation glycogen in small amounts of tissue, Anal. Biochem. 11 (1965), 256-265.

[13] M. Sok, M. Sentjure and M. Schara, Membrane fluidity characteristics of human lung cancer, Cancer Lett. 139 (1999), 215-220.

[14] Y. Liu, R.-K. Cho, K. Sakurai, T. Miura and Y. Ozaki, Structures on spectra/structure correlations in near-infrared spectra of proteins and polypeptides, Part 1: A marker band for hydrogen bonds, Appl. Spectrosc. 48 (1994), 1249-1254. 


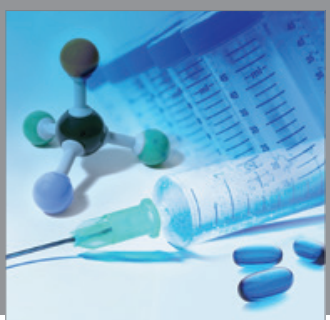

International Journal of

Medicinal Chemistry

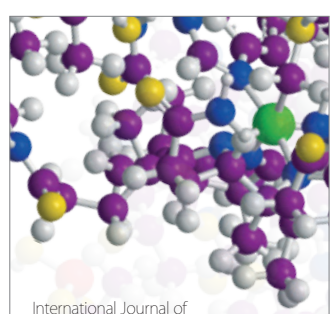

Carbohydrate Chemistry

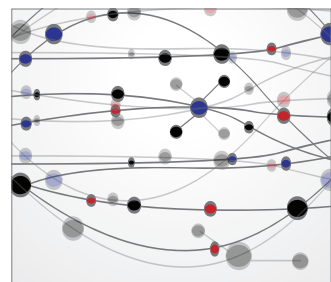

The Scientific World Journal
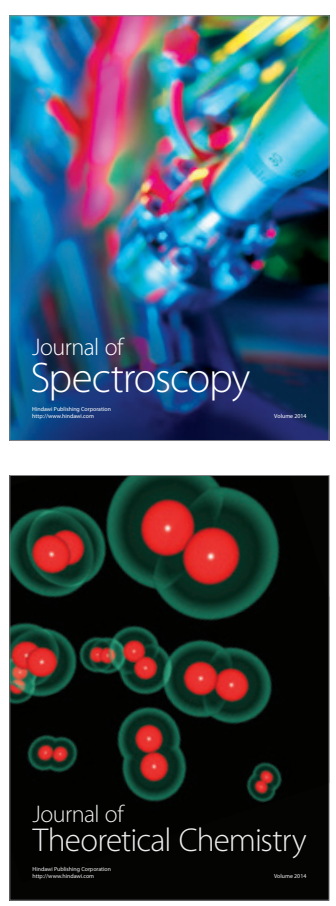
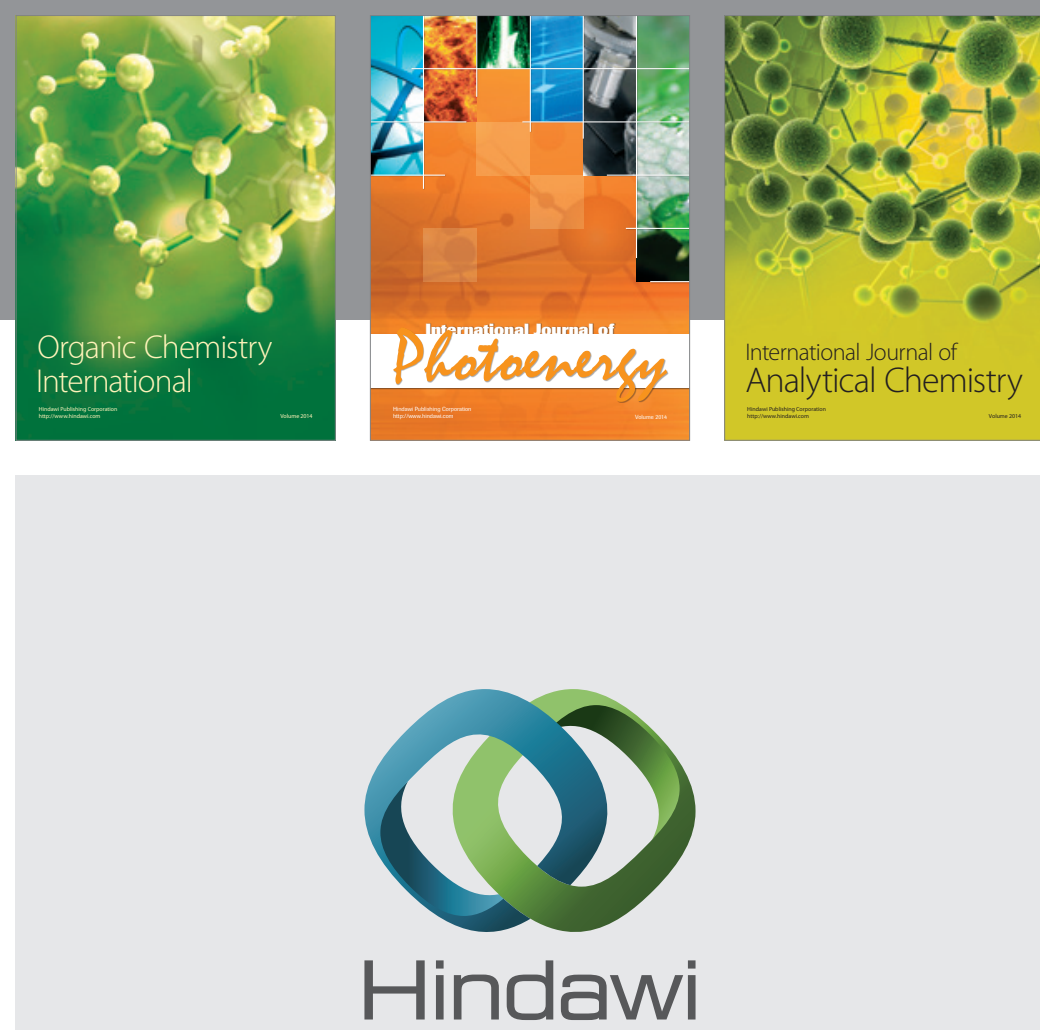

Submit your manuscripts at

http://www.hindawi.com
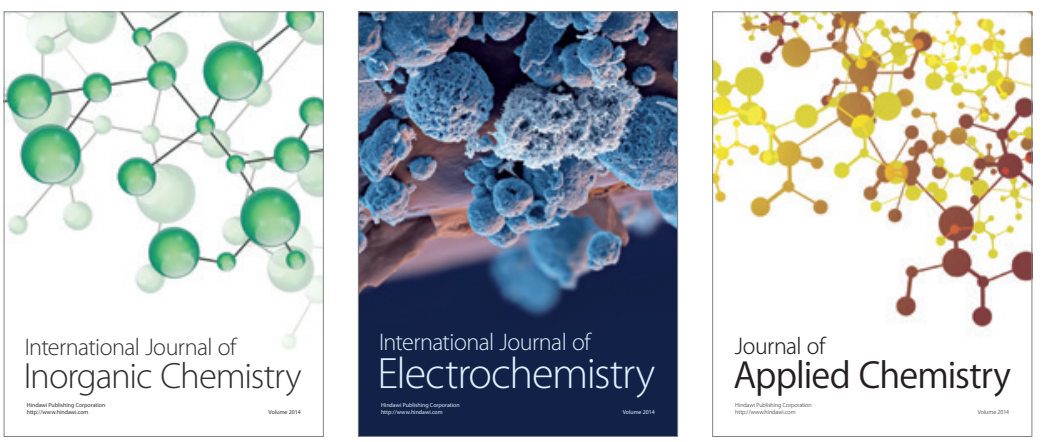

Journal of

Applied Chemistry
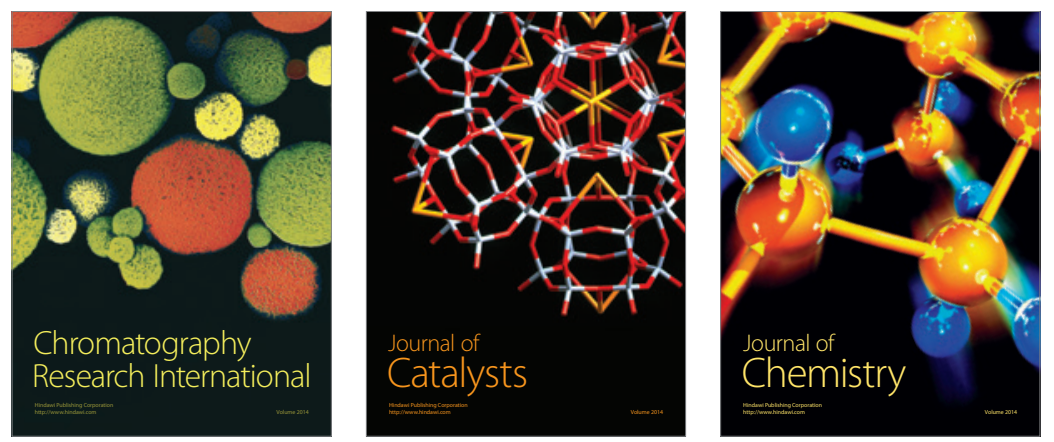
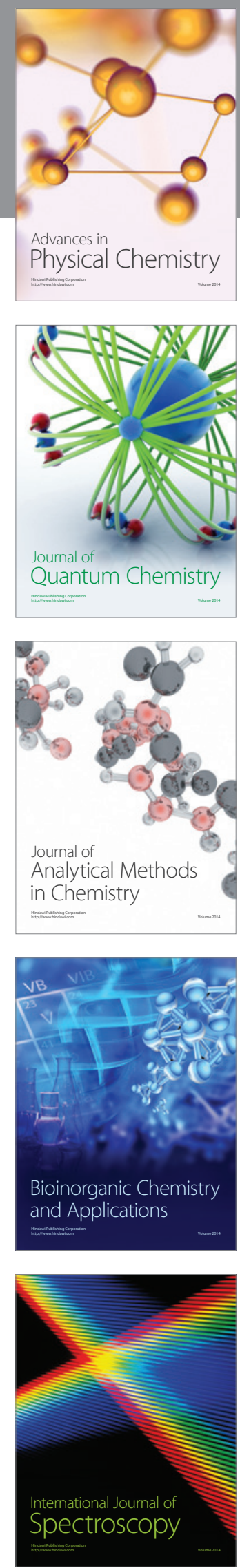